foot and toes. During an episode, which lasted for one to five seconds, the patient complained of sharp pain in the left extrem ities that could not be explained solely by the muscle contraction causing the dystonic posturing. There were no alteration of consciousness, loss of sphincter control, or clonic movements in the limbs. The attacks had started without obvious precipitating factors 10 days before admission and occurred with a frequency of 5-10 per day.

The patient reported that at the age of 23 she had experienced a single similar attack of "painful spasm" in her left extremities that had not been treated and had not recurred.

Mild depressive symptoms had been present since the age of 60 , being treated with fluvoxamine and benzodiazepines intermittently. There was no family history of epilepsy or other neurological diseases.

Her interictal general and neurological examination was unremarkable. Blood counts and serum biochemistry were normal. The routine EEG recording was normal. After sleep deprivation the awake EEG disclosed no abnormalities; during drowsiness and sleep an almost continuous focal epileptic activity was apparent in the right frontal-frontal sagittal region (figure) Computed tomography with and without contrast and MRI of the brain were performed and showed mild symmetric brain atrophy without any evidence of focal structural lesions or changes suggestive of demyelinating disease. Treatment with carbamazepine (200 $\mathrm{mg}$ three times daily) led to dramatic cessation of attacks and disappearance of the epileptic activity on the EEG.

Painful paroxysmal dystonia was previously described by different terms, such as painful tonic seizures and painful tonic spasm. ${ }^{\prime}$ It represents one of the distinct paroxysmal features of multiple sclerosis. Ephaptic activation of axons within demyelinated plaques or other structural lesion may be responsible for both motor and sensory components of the paroxysms.

Bennett $^{2}$ attributed painful paroxysmal dystonia to the heterogeneous group of paroxysmal dyskinesiae, stressing their nonepileptic character and emphasising their important place in the differential diagnosis of epilepsy. The most characteristic features of these fits are their painful nature and typical pattern of unilateral limb posturing. ${ }^{12}$ Both ictal and interictal EEGs were reported to be normal or showed non-specific changes ${ }^{12}$ supporting the non-epileptic origin of painful paroxysmal dystonia. By contrast, dystonic posturing may occur in epilepsy, albeit it is usually not painful. ${ }^{3}$

Paroxysmal pain associated with motor phenomena may also be a rare manifestation of epilepsy. ${ }^{4}$ It has been suggested that epileptic pain originates from the contralateral rolandic area ${ }^{4}$ or superior and medial parts of the parietal lobe. ${ }^{5}$ The motor component of painful epileptic seizures was reported to be represented by unilateral clonic or tonic-clonic convulsions with or without march, tonic deviation of the head and eyes, bilateral clonic movements of the extremities, generalised tonic-clonic convulsions, transient motor weakness without tonic or clonic phenomena, and "stiffness" of the arm or leg. ${ }^{45}$ Painful epileptic seizures with a characteristic pattern of unilateral posturing of the extremities meeting the criteria of painful paroxysmal dystonia to our knowledge have apparently never been described.

Our patient had typical unprovoked painful paroxysmal dystonia associated with focal cortical epileptic activity in the contralateral frontal-frontal sagittal region. This approximates to the premotor and supplementary motor region that, when activated, may result in postural changes. ${ }^{2}$

Although we have not obtained an ictal recording, the association of the clinical events with a very active epileptic focus in the EEG and the disappearance of both after carbamazepine treatment, suggest a causal relation between the electrographic phenomena and the bouts supporting thei epileptic nature. They may represent a distinct type of painful paroxysmal dystonia of cortical epileptic origin.

F BOKSTEIN $M$ Y NEUFELD P NISIPEANU A D Department of Neurology, Tel Aviv Medical Center

Sackler Faculty of Medicine,
Tel Aviv University, Israe

Correspondence to: Dr Felix Bokstein, Department of Neurology, Tel Aviv Souraski Medica

1 Osterman PO, Westberg CE. Paroxysmal attacks in multiple sclerosis. Brain 1975;98: 198-202.

2 Bennett DA. Paroxysmal dyskinesias. In: Resor SR, ed. The medical treatment of Resor SR, ed. The medical treatment of 1992:219-21.

3 Korczyn AD, Inzelberg R. Dystonia. Cur Opin Neurol Neurosurg 1993;6:350-7.

4 Young GB, Blume WT. Painful epileptic seizures. Brain 1983;106:537-54.

5 Wilkinson HA. Epileptic pain. An uncommon manifestation with localizing value Neurology 1973;23:518-20.

Treatment of spasmodic torticollis with intramuscular phenol injection

Phenol is a caustic agent that produces tissue destruction and has been used to weaken muscle in patients with spasticity by either nerve block, motor point block, or intramuscular neurolysis. ${ }^{1-5}$ Local muscle pain and tenderness but no systemic or long term side effects have been reported after such use. There is no report of the use of intramuscular phenol in the treatment of spasmodic torticollis. I have used this agen in patients with spasmodic torticollis who have not responded well to intramuscular injections of botulinum toxin A (BTX) Both patients gave informed consent to participate in the trial with the approval of the Duke University Investigational Review Board.

The muscles responsible for abnormal head movements were determined by clinical examination and by EMG recordings made with a concentric EMG needle in the sternomastoid, splenius capitus, or other neck muscles involved in head turning Phenol solution ( $1 \%$ weight/volume USP phenol crystals in sterile aqueous solution) was injected into the involved muscles with a recording monopolar injection electrode to determine that injections were made in muscle active in the abnormal movement. Injections were made at two to six sites in each muscle, in sites where motor unit action potentials with sharply rising components were recorded, indicating that the needle tip was close to muscle fibres that were activated during the abnormal move- ment. Areas near major vessels or nerves were avoided. After 18 hours or more, subsequent injections were given if improvement was incomplete as determined by the patient's symptoms and by examination. At each visit, examination of strength in the involved muscles and functional assessment of the patient's torticollis were recorded on videotape for comparison with examinations made before phenol treatment.

Patient 1 is a 53 year old man who has had torticollis since the age of 43. Medical treatment had produced no improvement. At the age of 48 , he had shown moderate improvement with BTX injections but, after 18 months, the response to repeated injections diminished despite increasing doses. Muscles no longer developed weakness, atrophy, or denervation changes on EMG testing after BTX was injected. Antibody to botulinum could not be detected in the serum. He underwent selective peripheral denervation surgery when aged 50 and 51 , with only mild improvement. Several months later, BTX injections were repeated without benefit.

$\mathrm{He}$ had tonic, uncontrollable turning of the head to the left with a phasic component and mild retrocollis before phenol injection. He maintained his head in the midline with great difficulty while sitting. Standing and any attempt to talk, walk, or use his hands produced immediate uncontrollable head turning. He had constant posterior neck and intrascapular pain.

Within 18 hours after the initial injection of $100 \mathrm{mg}$ phenol into the left splenius capitis, splenius cervicis, and longissimus capitus muscles, he noted a fuller range of motion in the neck and mild improved head control while walking. The injected muscles were tender and oedematous but the neck and intrascapular pain was considerably less. After subsequent injections he noted progressive improvement. After receiving a total dose of $500 \mathrm{mg}$ of phenol over one month, he could walk, drive, or sit to eat even in public with only occasional involuntary head turning. Manoeuvres that previously had exacerbated the abnormal head movements now were performed with little or no difficulty. He estimated that pain was reduced by $90 \%$. His only side effects were mild erythema and tenderness over the injected muscles lasting one to two days after each injection. The improvement was sustained for five months after the initial injection. $\mathrm{He}$ then experienced gradual worsening of head control and some return of pain. He has subsequently received phenol injections at intervals of six months to maintain improvement.

Patient 2 is a 43 year old white male physician who had onset of involuntary head turning to the left at the age of 33 Medical treatment produced intolerable side effects and no appreciable improvement. At the age of 41 , he received BTX injections every three to four months with moderate improvement but symptoms of head turning and pain returned four to six weeks after each injection. After each BTX injection, he developed severe dysphagia that persisted for two weeks. Before pheno was injected, he had moderately severe torticollis that was greatly exacerbated by public speaking and manual activities.

Eighteen hours after the initial injection of $120 \mathrm{mg}$ phenol into the left splenius capitis, splenius cervicis, and longissimus capitus muscles, he noted improvement in neck 
pain but little change in head control. After subsequent injections of $260 \mathrm{mg}$ given over two days, he noted considerable improvement in the ability to drive and carry out other manual tasks in his work. He graded improvement in head control and pain as $50 \%$ and $80 \%$, respectively, above baseline, compared with $50 \%$ and $50 \%$ after the previous BTX injections. He had transient mild tenderness in the injected muscles. After the first month he experienced a slight decline $(10 \%)$ in function and pain control that remained constant for the next four months and then gradually declined again. With subsequent injections of phenol at about six monthly intervals, he has maintained his maximal level of improvement.

These two patients had moderately severe spasmodic torticollis that had improved only partially after previous treatment. In the first patient, BTX initially provided relief but became ineffective. In the second patient, BTX provided improvement but the side effect of dysphagia was nearly intolerable. Within 18 hours after phenol injections into cervical muscles, there was definite reduction of involuntary movements and pain, with functional improvement. Improvement was greater than after all previous treatments and persisted for six and five months respectively, after the initial series of phenol injections. The only side effect was transitory-namely, mild tenderness in the injected muscles.

In patients who become resistant to repeated injections of BTX, presumably due to formation of antibody to the toxin, it would be of great benefit to have an alternative treatment. Phenol may be of benefit in this situation and has the additional advantage of being inexpensive. If the promising response in these two patients is confirmed in a larger series of patients I am currently studying, EMG guided intramuscular phenol injections may prove to be an effective treatment for some patients with spasmodic torticollis.

Division of Neurology, JANICE M MASSEY Department of Medicine, North Carolina, USA

Correspondence to: Dr Janice M Massey, Box 3403, Duke University Medical Center, Durham, North Carolina 27710, USA.

1 DeLateur BJ. A new technique of intramuscular phenol neurolysis. Arch Phys Med Muscular phenol neurolysis

2 Easton JK, Ozel T, Halpern D. Intramuscular neurolysis for spasticity in children. Arch Phys Med Rehabil 1979;60:155-8.

3 Garland DE, Lilling $M$, Keenan MA. Percutaneous phenol blocks to motor points of spastic forearm muscles in head-injured adults. Arch Phys Med Rehabil 1984;65: 243-5.

4 Gibson II. Phenol block in the treatment of spasticity. Gerontology 1987;33:327-30.

5 Keenan MAE. Management of the spastic upper extremity in the neurologically 233:116-25.

\section{Tuberculous myelopathy: a serial MRI} study

In two definitive publications in 1969 Wadia and Dastur delineated spinal meningitides with associated radiculomyelopathy with particular reference to tuberculosis. ${ }^{12}$ The advent of MRI has meant that the nature of the intramedullary lesions can for the first time be defined during life. Furthermore, the natural history of spinal

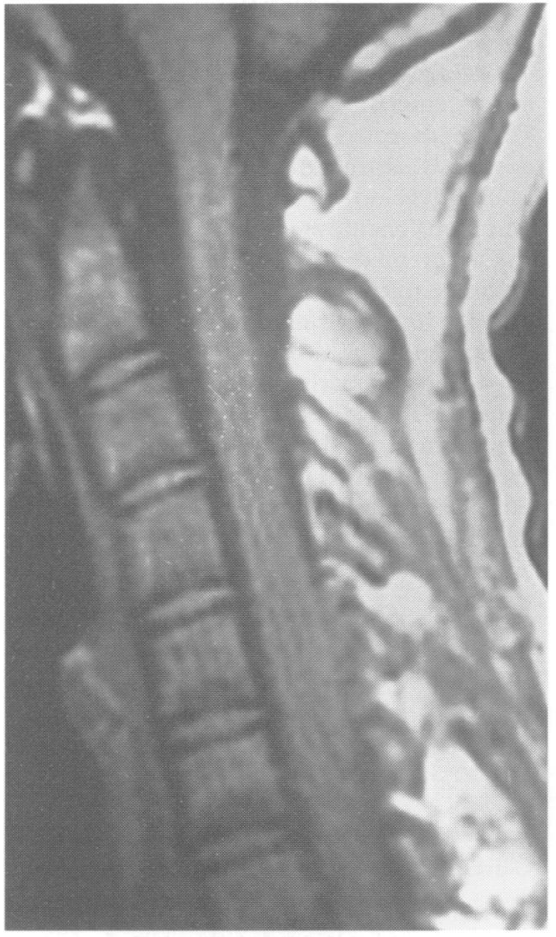

(A)

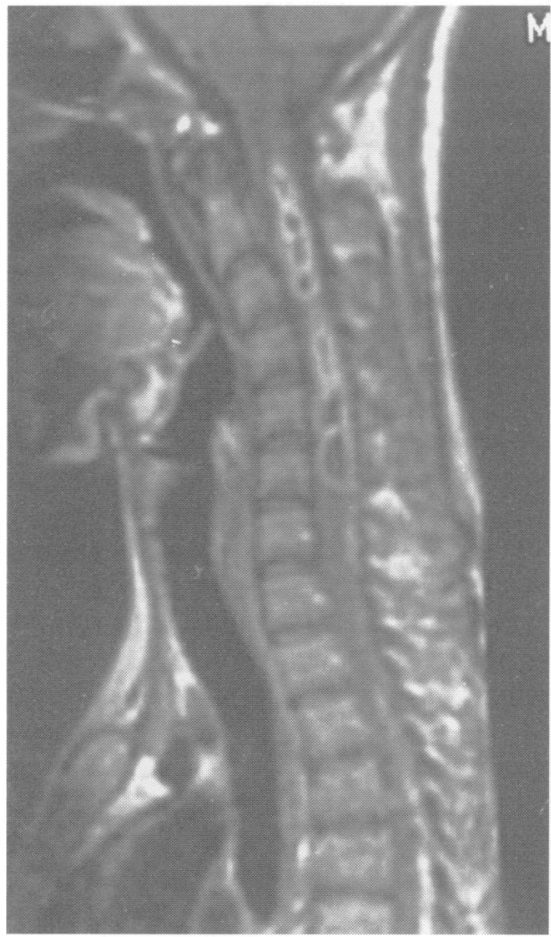

(C)

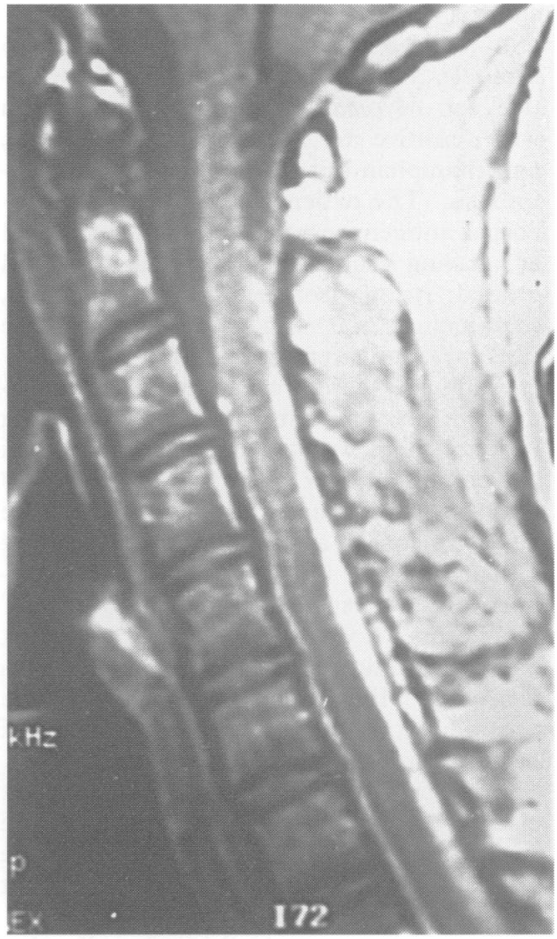

(B)

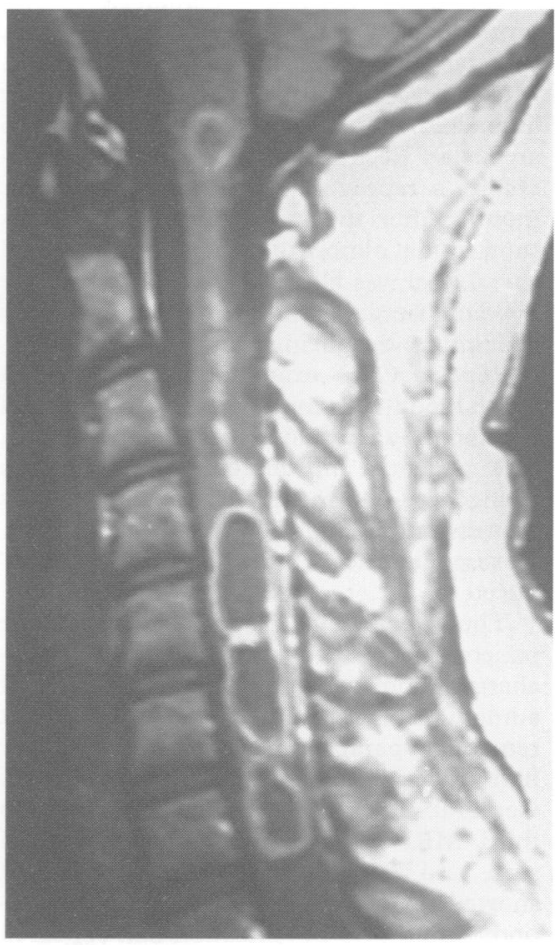

(D)

(A) Sagittal T1 weighted MRI of the cervical spine before and (B) after gadolinium-DTPA showing enhancement in the spinal meninges. (C) Sagittal T1 weighted MRI of cervical and dorsal spine after gadolinium - DTPA four months after the initial scan. Multiple gadolinium ring enhancing lesions are seen within the cervical cord. (D) Sagittal T1 weighted MRI of cervical cord showing further changes in the intramedullary cavities eight months after the initial scan. Intense gadolinium enhancement continues in some loculi, others have resolved, and a new locule has appeared at the cervicomedullary junction.

cord involvement in tuberculosis can be studied with serial MRI. The current study involves a single case followed up with a series of MRI examinations over an eight month period. It shows a previously unpublished pattern of cord involvement.

A 26 year old Samalian male refugee who had been in the United Kingdom for one year presented with a three month history of nausea and vomiting associated with increasingly severe headaches. He had no previous history of or exposure to tuberculosis. On admission to hospital he had neck stiffness only. Initial CSF examination 